\title{
Synthesis and biological evaluation of some new pyrimidines via a novel chalcone series
}

\author{
Amit R.Trivedi, Dipti K. Dodiya, Naresh R. Ravat, and Viresh H. Shah* \\ Department of Chemistry, Saurashtra University, Rajkot (Gujarat), India, Pin-360005 \\ E-mail: shahvh@yahoo.com
}

\begin{abstract}
In the present investigation ethyl 2-(4-carboxyphenylazo)acetoacetate $\mathbf{1}$ on condensation with various aromatic aldehydes in ethanolic $\mathrm{NaOH}$ solution yielded the corresponding chalcones 2a-j. These chalcones were further reacted with urea in the presence of base in ethanol, which led to the formation of pyrimidine derivatives $\mathbf{3 a - j}$. The newly synthesized heterocyles were characterized on the basis of their chemical properties and spectroscopic data. All newly synthesized compounds were evaluated for their antimycobacterial activities against Mycobacterium tuberculosis $\mathrm{H}_{37} \mathrm{Rv}$.
\end{abstract}

Keywords: Pyrimidine, antimycobacterial, Mycobacterium tuberculosis

\section{Introduction}

Tuberculosis (TB) is by far the most frequently encountered mycobacterial disease in the world. ${ }^{1}$ Although its incidence has diminished significantly in the industrially more developed countries; it remains a major public health problem in most of the developing nations. Tuberculosis is still the single largest infection having a high mortality rate and 0.1 to 0.3 percent of the population become infected each year in the developed countries. This year, 2 million people may develop the disease and 30 million may die worldwide (as per a WHO report). It is commonly known that Mycobacterium tuberculosis has developed resistance to the majority of the existing drugs. However, powerful new anti-TB drugs with new mechanisms of action have not been developed in the last forty years. In the developing countries, the annual infection rate is 20-50 times greater than in the developed countries and its high level shows little or no downward trend. It is expected that development of new effective anti-TB drugs will bring various outcomes viz: shortening the total duration of therapy, reducing the total expenditure and treatment of multiple drug resistant tuberculosis (MDR-TB) by single dosage regiment. ${ }^{2,3}$ In pursuit of achieving this goal, our research efforts are focused on the development of novel structural moieties having antimycobacterial properties. ${ }^{4,5}$ Chalcones have various biological activities such as cytotoxic, ${ }^{5}$ 
antimalarial, ${ }^{6}$ antioxidant, ${ }^{7}$ tyrosinase Inhibitory, ${ }^{8}$ anti-inflammatory, ${ }^{9}$ cancer chemopreventive ${ }^{9}$ and antibacterial. ${ }^{10}$ Several pyrimidine derivatives have wide varieties of usages and its nucleus is also present in vitamin $\mathrm{B}_{2}$ and folic acid. Pyrimidine heterocycles possessing hydroxyl group has a unique place in medicinal chemistry, ${ }^{11}$ and also plays a vital role in biological processes ${ }^{12}$, ${ }^{13}$ as well as synthetic drugs. ${ }^{14}$ Pyrimidines are associated with various therapeutic activities e.g., anti-HIV, ${ }^{15}$ anti-tubercular, ${ }^{16}$ antitumor, ${ }^{17}$ antineoplastic, ${ }^{18}$ anti-inflammatory, ${ }^{19}$ diuretic, ${ }^{20}$ antimalaria, ${ }^{21}$ cardiovascular. ${ }^{22}$ To the best of our knowledge, there has been no previous reports of analogous pyrimidines and parent chalcones as antituberculosis agents. However, there are numerous examples of nitrogen containing heterocycles being used to treat TB, for example Clofazimine, Isoniazid and Pyrazinamide. These compounds provide structural precedence that our chalcone and pyrimidine analogues may lead to the generation of novel anti-TB therapeutics. Herein the synthesis and in vitro antimycobacterial activity of novel chalcone and pyrimidine derivatives are described.

\section{Results and Discussion}

\section{Chemistry}

The synthesis of chalcone and pyrimidine derivatives was performed following the steps shown in Scheme-1. In the initial step, chalcones (2a-j) were synthesized by condensing 2-(4carboxyphenylazo)acetoacetate $^{23} \mathbf{1}$ with appropriate arometic aldehydes in dilute ethanolic sodium hydroxide solution at room temperature. The compounds (3a-j) were synthesized by reacting the appropriate chalcone with urea and potassium hydroxide in ethanol. The purity of the compounds was determined by TLC and elemental analysis. Spectral data (IR, ${ }^{1} \mathrm{H}-\mathrm{NMR},{ }^{13} \mathrm{C}$ NMR and Mass) of all the newly synthesized compounds were in full agreement with the proposed structures. 

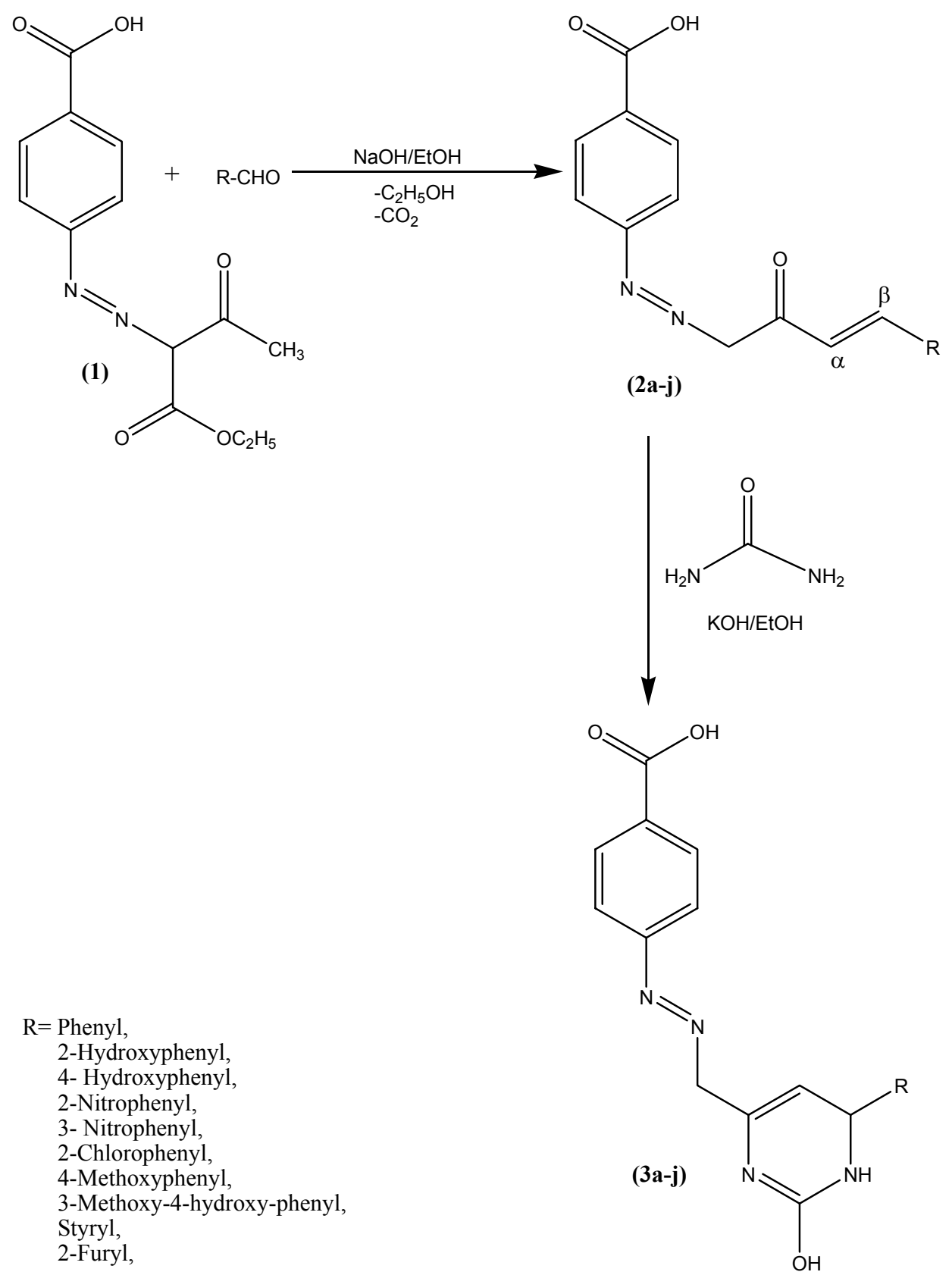

\section{Scheme 1}

The possible mechanism involved in the formation of dihydropyrimidine derivatives (3a-j) from the respective chalcones (2a-j) is shown below. 

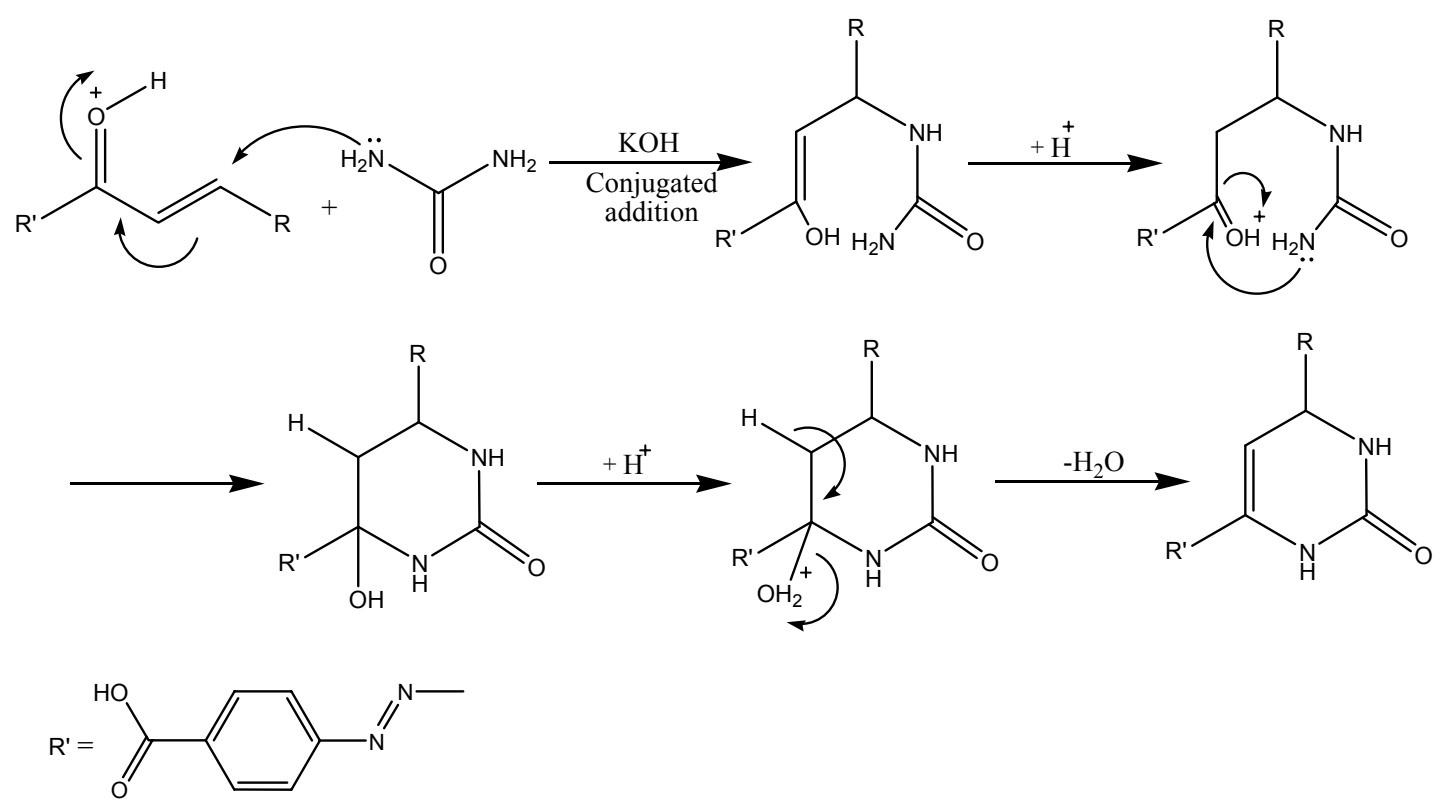

\section{Biological screening}

\section{Microbiology}

The in vitro activities of the synthesized compounds (2a-j \& 3a-j) for tuberculosis inhibition against the Mycobacterium tuberculosis $\mathrm{H}_{37} \mathrm{Rv}$ (ATCC27294) strain were performed using the micro plate alamar blue assay (MABA) $)^{24}$ method at TAACF. Compounds exhibiting fluorescence are tested in a BACTEC-460 radiometric system ${ }^{25,26}$ and/or broth micro dilution assay. The activities are expressed as minimum inhibitory concentration (MIC, $\mu \mathrm{g} / \mathrm{mL}$ ) and are summarized in Table 1. Compounds demonstrating at least $90 \%$ inhibition were re-tested at lower concentrations to determine the actual MIC, a value defined as the lowest concentration inhibiting $\approx 90 \%$ of the inoculums relative to the control.

\section{Antimycobacterial activity}

Twenty compounds were screened for their antimycobacterial activity against Mycobacterium tuberculosis $\mathrm{H}_{37} \mathrm{Rv}$ using a BACTEC-460 radiometric system. Among the chalcones 2a-j and pyrimidines $\mathbf{3 a - j}$, compounds $\mathbf{3 d}, \mathbf{3 e}$ and $\mathbf{3 g}$ produced the highest efficacy and exhibited $>90 \%$ inhibition at $6.25 \approx \mu \mathrm{g} / \mathrm{ml}$ in the primary screen (Table 1). Compounds $\mathbf{2 a - j}, \mathbf{3 a - c}, \mathbf{3 f}$ and $\mathbf{3 h}-\mathbf{j}$ exhibited $>90 \%$ inhibition against Mycobacterium tuberculosis at $6.25 \mu \mathrm{g} / \mathrm{mL}$ (Table 1). These antimycobacterial data clearly indicated that the presence of 2-nitro, 3-nitro and 4-methoxy substitution on chalcone produced remarkable improvements in antitubercular activity. 
Table 1. In vitro antimycobacterial screening data of $\mathbf{2 a - j} \& \mathbf{3} \mathbf{a}-\mathbf{j}$

\begin{tabular}{cllcc}
\hline Sr. & R & Molecular & MIC & Inhibition \\
No. & & Formula & $\mu \mathrm{g} / \mathrm{mL}$ & \\
\hline 2a & Phenyl & $\mathrm{C}_{17} \mathrm{H}_{14} \mathrm{~N}_{2} \mathrm{O}_{3}$ & $<6.25$ & - \\
2b & 2-Hydroxyphenyl & $\mathrm{C}_{17} \mathrm{H}_{14} \mathrm{~N}_{2} \mathrm{O}_{4}$ & $<6.25$ & 10 \\
2c & 4- Hydroxyphenyl & $\mathrm{C}_{17} \mathrm{H}_{14} \mathrm{~N}_{2} \mathrm{O}_{4}$ & $<6.25$ & 20 \\
2d & 2-Nitrophenyl & $\mathrm{C}_{17} \mathrm{H}_{13} \mathrm{~N}_{3} \mathrm{O}_{5}$ & $<6.25$ & 56 \\
2e & 3- Nitrophenyl & $\mathrm{C}_{17} \mathrm{H}_{13} \mathrm{~N}_{3} \mathrm{O}_{5}$ & $<6.25$ & 50 \\
2f & 2-Chlorophenyl & $\mathrm{C}_{17} \mathrm{H}_{13} \mathrm{~N}_{2} \mathrm{O}_{3} \mathrm{Cl}$ & $<6.25$ & 25 \\
2g & 4-Methoxyphenyl & $\mathrm{C}_{18} \mathrm{H}_{16} \mathrm{~N}_{2} \mathrm{O}_{4}$ & $<6.25$ & 28 \\
2h & 3-Methoxy-4-hydroxy-phenyl & $\mathrm{C}_{18} \mathrm{H}_{16} \mathrm{~N}_{2} \mathrm{O}_{5}$ & $<6.25$ & 38 \\
2i & Styryl & $\mathrm{C}_{19} \mathrm{H}_{16} \mathrm{~N}_{2} \mathrm{O}_{3}$ & $<6.25$ & 18 \\
2j & 2-Furyl & $\mathrm{C}_{15} \mathrm{H}_{12} \mathrm{~N}_{2} \mathrm{O}_{4}$ & $<6.25$ & - \\
3a & Phenyl & $\mathrm{C}_{18} \mathrm{H}_{16} \mathrm{~N}_{4} \mathrm{O}_{3}$ & $<6.25$ & 90 \\
3b & 2-Hydroxyphenyl & $\mathrm{C}_{18} \mathrm{H}_{16} \mathrm{~N}_{4} \mathrm{O}_{4}$ & $>6.25$ & 92 \\
3c & 4- Hydroxyphenyl & $\mathrm{C}_{18} \mathrm{H}_{16} \mathrm{~N}_{4} \mathrm{O}_{4}$ & $<6.25$ & 87 \\
3d & 2-Nitrophenyl & $\mathrm{C}_{18} \mathrm{H}_{15} \mathrm{~N}_{5} \mathrm{O}_{5}$ & $>6.25$ & 96 \\
3e & 3- Nitrophenyl & $\mathrm{C}_{18} \mathrm{H}_{15} \mathrm{~N}_{5} \mathrm{O}_{5}$ & $>6.25$ & 96 \\
3f & 2-Chlorophenyl & $\mathrm{C}_{18} \mathrm{H}_{15} \mathrm{~N}_{4} \mathrm{O}_{3} \mathrm{Cl}$ & $>6.25$ & 92 \\
3g & 4-Methoxyphenyl & $\mathrm{C}_{19} \mathrm{H}_{18} \mathrm{~N}_{4} \mathrm{O}_{4}$ & $>6.25$ & 97 \\
3h & 3-Methoxy-4-hydroxy-phenyl & $\mathrm{C}_{19} \mathrm{H}_{18} \mathrm{~N}_{4} \mathrm{O}_{5}$ & $<6.25$ & 90 \\
3i & Styryl & $\mathrm{C}_{20} \mathrm{H}_{18} \mathrm{~N}_{4} \mathrm{O}_{3}$ & $<6.25$ & 81 \\
3j & 2-Furyl & $\mathrm{C}_{16} \mathrm{H}_{14} \mathrm{~N}_{4} \mathrm{O}_{4}$ & $<6.25$ & 75 \\
\hline
\end{tabular}

\section{Conclusions}

In conclusion, a new class of chalcone and pyrimidines derivatives were synthesized and evaluated as antitubercular agents. The newly synthesized heterocycles exhibited promising antitubercular activities against Mycobacterium tuberculosis. These results makes novel chalcone and pyrimidine derivatives interesting lead molecules for further synthetic and biological evaluation. It can be concluded that this class of compounds certainly holds great promise towards the pursuit to discover novel classes of antimycobacterial agents. Further studies to acquire more information concerning structure-activity relationships are in progress.

\section{Experimental Section}

General Procedures. Chemicals were supplied by E. Merck (Germany) and S. D. Fine Chemicals (India). Melting points were determined by the open tube capillary method and are 
uncorrected. The purity of the compounds was monitored by thin layer chromatography (TLC) plates (silica gel $\mathrm{G}$ ) in the solvent system toluene-ethyl formate-formic acid (5:4:1) and benzene-methanol (8:2). The spots were observed by exposure to iodine vapours or by UV light. The IR spectra were obtained on a Perkin-Elmer 1720 FT-IR spectrometer (KBr pellets). The ${ }^{1} \mathrm{H}-$ NMR spectra were recorded on a Bruker AC $300 \mathrm{MHz}$ spectrometer using TMS as the internal standard in DMSO- $d_{6} / \mathrm{CDCl}_{3} \cdot{ }^{13} \mathrm{C}-\mathrm{NMR}(75$ and $125 \mathrm{MHz})$ spectra were measured on a Bruker $\mathrm{AC} 200$, DPX 300 and $\mathrm{ARX} 500$, at $25^{\circ} \mathrm{C}$, in $\mathrm{CDCl}_{3}$. Elemental analyses of the newly synthesized compounds were carried out on Carlo Erba 1108 analyzer.

General procedure for the synthesis of 3-(4'-aryl)-1-[(4)-carboxyphenylazomet hyl]-2-propene-1-one (2a-j)

To a mixture of ethyl 2-(4-carboxyphenylazo)acetoacetate (1) $(0.01 \mathrm{~mol})$ and the appropriate aldehyde $(0.01 \mathrm{~mol})$ in oxygen-free ethanol was added a solution of sodium hydroxide in oxygen-free distilled water with constant stirring of the reaction flask. The reaction mixture was stirred for 24 hours on a magnetic stirrer and poured on to crushed ice. The solid mass that separated out was filtered, washed with water and crystallized from ethanol to furnish the desired product (2a-j) as yellow crystals.

3-(4'-Phenyl)-1-[(4)-carboxyphenylazomethyl]-2-propene-1-one (2a). Yield 69\%, mp. 156$158{ }^{\circ} \mathrm{C}$, IR (KBr): $1480(\mathrm{~N}=\mathrm{N}), 1682(\mathrm{C}=\mathrm{O}), 1713\left(\mathrm{C}=\mathrm{O}\right.$ of acid), 3035-3042 (CH); ${ }^{1} \mathrm{H}-\mathrm{NMR}$ (DMSO-d $)): \delta 3.00\left(\mathrm{~s}, 2 \mathrm{H}, \mathrm{CH}_{2}\right), 6.67\left(\mathrm{~d}, 1 \mathrm{H}, \mathrm{H}_{\alpha}\right), 6.98\left(\mathrm{~d}, 1 \mathrm{H}, \mathrm{H}_{\beta}\right), 7.14-8.10(\mathrm{~m}, 9 \mathrm{H}$, aromatic), $11.00(\mathrm{~s}, 1 \mathrm{H}, \mathrm{OH}) ;{ }^{13} \mathrm{C}-\mathrm{NMR}\left(\mathrm{CDCl}_{3}\right): \delta 58.2,123.4,126.5,126.8,127.9,128.2$, 128.9, 130.9, 136.2, 143.2, 157.2, 170.2, 197.5. Mass $(\mathrm{m} / \mathrm{z})$ : 284. Anal. (\%) for $\mathrm{C}_{15} \mathrm{H}_{12} \mathrm{~N}_{2} \mathrm{O}_{4}$, Calcd. C, 63.38; H, 4.25; N, 9.85; O, 22.51; Found: C, 63.27; H, 4.12; N, 9.75; O, 22.40.

3-(2'-Hydroxyphenyl)-1-[(4)-carboxyphenylazomethyl]-2-propene-1-one (2b). Yield 74\%, mp. 196-198 ${ }^{\circ} \mathrm{C}$, IR (KBr): $1483(\mathrm{~N}=\mathrm{N}), 1676(\mathrm{C}=\mathrm{O}), 1707(\mathrm{C}=\mathrm{O}$ of acid), 3035-3042 $(\mathrm{CH})$, $3253(\mathrm{OH}) ;{ }^{1} \mathrm{H}-\mathrm{NMR}\left(\mathrm{DMSO}-d_{6}\right): \delta 3.03\left(\mathrm{~s}, 2 \mathrm{H}, \mathrm{CH}_{2}\right), 10.16(\mathrm{~s}, 1 \mathrm{H}, \mathrm{Ar}-\mathrm{OH}), 6.35\left(\mathrm{~d}, 1 \mathrm{H}, \mathrm{H}_{\alpha}\right)$, $6.93\left(\mathrm{~d}, 1 \mathrm{H}, \mathrm{H}_{\beta}\right), 7.18-8.10\left(\mathrm{~m}, 8 \mathrm{H}\right.$, aromatic), $11.03(\mathrm{~s}, 1 \mathrm{H}, \mathrm{OH}) ;{ }^{13} \mathrm{C}-\mathrm{NMR}\left(\mathrm{CDCl}_{3}\right): \delta 58.7$, $115.9,116.8,122.3,122.9,126.7,127.9,128.1,130.2,131.1,143.4,156.7,158.9,170.1,197.2$. Mass (m/z): 310. Anal. (\%) for $\mathrm{C}_{17} \mathrm{H}_{14} \mathrm{~N}_{2} \mathrm{O}_{4}, \mathrm{C}, 65.80 ; \mathrm{H}, 4.55 ; \mathrm{N}, 9.03$; O, 20.62; Found: C, $65.69 ; \mathrm{H}, 4.43 ; \mathrm{N}, 8.91 ; \mathrm{O}, 20.50$.

3-(4'-Hydroxyphenyl)-1-[(4)-carboxyphenylazomethyl]-2-propene-1-one (2c). Yield 70\%, mp. 170-172 ${ }^{\circ} \mathrm{C}$, IR (KBr): $1484(\mathrm{~N}=\mathrm{N}), 1689(\mathrm{C}=\mathrm{O}), 1705(\mathrm{C}=\mathrm{O}$ of acid), $3039(\mathrm{CH}), 3353$ $(\mathrm{OH}) ;{ }^{1} \mathrm{H}-\mathrm{NMR}\left(\mathrm{DMSO}-d_{6}\right): \delta 3.10\left(\mathrm{~s}, 2 \mathrm{H}, \mathrm{CH}_{2}\right), 5.10(\mathrm{~s}, 1 \mathrm{H}, \mathrm{Ar}-\mathrm{OH}), 6.44\left(\mathrm{~d}, 1 \mathrm{H}, \mathrm{H}_{\alpha}\right), 6.93(\mathrm{~d}$, $\left.1 \mathrm{H}, \mathrm{H}_{\beta}\right), 7.08-7.54\left(\mathrm{~m}, 8 \mathrm{H}\right.$, aromatic), $11.03(\mathrm{~s}, 1 \mathrm{H}, \mathrm{OH}) ;{ }^{13} \mathrm{C}-\mathrm{NMR}\left(\mathrm{CDCl}_{3}\right): \delta$ 58.3, 116.2, 122.9, 126.6, 127.5, 128.1, 131.1, 143.4, 156.7, 157.9, 170.3, 198.1. Mass (m/z): 310. Anal. (\%) for $\mathrm{C}_{17} \mathrm{H}_{14} \mathrm{~N}_{2} \mathrm{O}_{4}$, Calcd. C, 65.80; H, 4.55; N, 9.03; O, 20.62; Found: C, 65.67; H, 4.41; N, 8.92; $\mathrm{O}, 20.52$.

3-(2'-Nitrophenyl)-1-[(4)-carboxyphenylazomethyl]-2-propene-1-one (2d). Yield 72\%, mp. 181-183 ${ }^{\circ} \mathrm{C}$, IR (KBr): $1365\left(\mathrm{NO}_{2}\right), 1489(\mathrm{~N}=\mathrm{N}), 1692(\mathrm{C}=\mathrm{O}), 1713(\mathrm{C}=\mathrm{O}$ of acid), $3039(\mathrm{CH})$; ${ }^{1} \mathrm{H}-\mathrm{NMR}\left(\mathrm{DMSO}-d_{6}\right): \delta 3.10\left(\mathrm{~s}, 2 \mathrm{H}, \mathrm{CH}_{2}\right), 6.78\left(\mathrm{~d}, 1 \mathrm{H}, \mathrm{H}_{\alpha}\right), 7.14\left(\mathrm{~d}, 1 \mathrm{H}, \mathrm{H}_{\beta}\right), 7.40-8.10(\mathrm{~m}, 8 \mathrm{H}$, 
aromatic), $11.20(\mathrm{~s}, 1 \mathrm{H}, \mathrm{OH}) ;{ }^{13} \mathrm{C}-\mathrm{NMR}\left(\mathrm{CDCl}_{3}\right): \delta 58.2,121.4,122.7,126.6,127.8,129.1$, 130.4, 131.1, 135.1, 143.2, 146.8, 156.9, 170.8, 197.8. Mass $(m / z)$ : 339. Anal. (\%) for $\mathrm{C}_{17} \mathrm{H}_{13} \mathrm{~N}_{3} \mathrm{O}_{5}$, Calcd. C, 60.18; H, 3.86; N, 12.38; O, 23.58; Found: C, 60.07; H, 3.73; N, 12.26; $\mathrm{O}, 23.46$.

3-(3'-Nitrophenyl)-1-[(4)-carboxyphenylazomethyl]-2-propene-1-one (2e). Yield 68\%, mp. 197-199 ${ }^{\circ} \mathrm{C}$, IR (KBr) $1363\left(\mathrm{NO}_{2}\right), 1485(\mathrm{~N}=\mathrm{N}), 1680(\mathrm{C}=\mathrm{O}), 1703(\mathrm{C}=\mathrm{O}$ of acid), $3042(\mathrm{CH})$; ${ }^{1} \mathrm{H}-\mathrm{NMR}\left(\mathrm{DMSO}-d_{6}\right): \delta 3.0\left(\mathrm{~s}, 2 \mathrm{H}, \mathrm{CH}_{2}\right), 6.84\left(\mathrm{~d}, 1 \mathrm{H}, \mathrm{H}_{\alpha}\right), 7.18\left(\mathrm{~d}, 1 \mathrm{H}, \mathrm{H}_{\beta}\right), 7.32-8.10(\mathrm{~m}, 8 \mathrm{H}$, aromatic), $11.10(\mathrm{~s}, 1 \mathrm{H}, \mathrm{OH}) ;{ }^{13} \mathrm{C}-\mathrm{NMR}\left(\mathrm{CDCl}_{3}\right): \delta 58.6,120.8,121.9,122.9,126.8,127.5$, $130.3,131.1,132.9,137.2,143.5,149.2,157.2,170.6,196.9$. Mass $(m / z): 310$. Anal. (\%) for $\mathrm{C}_{17} \mathrm{H}_{14} \mathrm{~N}_{2} \mathrm{O}_{4}, \mathrm{C}, 65.80$; H, 4.55; N, 9.03; O, 20.62; Found: C, 65.67; H, 4.43; N, 8.89; O, 20.51.

3-(2'-Chlorophenyl)-1-[(4)-carboxyphenylazomethyl]-2-propene-1-one (2f). Yield 71\%, mp. 200-202 ${ }^{\circ} \mathrm{C}$, IR (KBr): $760(\mathrm{Cl}), 1481(\mathrm{~N}=\mathrm{N}), 1689(\mathrm{C}=\mathrm{O}), 1715\left(\mathrm{C}=\mathrm{O}\right.$ of acid), $3043(\mathrm{CH}) ;{ }^{1} \mathrm{H}-$ NMR (DMSO-d $\left.)_{6}\right): \delta 3.12\left(\mathrm{~s}, 2 \mathrm{H}, \mathrm{CH}_{2}\right), 6.47\left(\mathrm{~d}, 1 \mathrm{H}, \mathrm{H}_{\alpha}\right), 6.87\left(\mathrm{~d}, 1 \mathrm{H}, \mathrm{H}_{\beta}\right), 7.08-8.10(\mathrm{~m}, 8 \mathrm{H}$, aromatic), $11.03(\mathrm{~s}, 1 \mathrm{H}, \mathrm{OH}) ;{ }^{13} \mathrm{C}-\mathrm{NMR}\left(\mathrm{CDCl}_{3}\right): \delta 58.0,123.0,124.9,126.7,126.9,127.8$, $128.4,130.6,131.1,135.2,137.2,143.5,157.4,171.1,197.9$. Mass $(m / z): 328$. Anal. (\%) for $\mathrm{C}_{17} \mathrm{H}_{13} \mathrm{ClN}_{2} \mathrm{O}_{3}, \mathrm{C}, 62.11 ; \mathrm{H}, 3.99 ; \mathrm{Cl}, 10.78 ; \mathrm{N}, 8.52 ; \mathrm{O}, 14.60$; Found: C, 62.00; H, 3.86; Cl, $10.63 ; \mathrm{N}, 8.41 ; \mathrm{O}, 14.46$.

3-(4'-Methoxyphenyl)-1-[(4)-carboxyphenylazomethyl]-2-propene-1-one (2g). Yield 70\%, mp. 203-205 ${ }^{\circ} \mathrm{C}$, IR (KBr): 1159 (C-O-C), $1488(\mathrm{~N}=\mathrm{N}), 1682(\mathrm{C}=\mathrm{O}), 1707$ (C=O of acid), 3039 $(\mathrm{CH}) ;{ }^{1} \mathrm{H}-\mathrm{NMR}\left(\mathrm{DMSO}-d_{6}\right): \delta 3.10\left(\mathrm{~s}, 2 \mathrm{H}, \mathrm{CH}_{2}\right), 3.73\left(\mathrm{~s}, 1 \mathrm{H}, \mathrm{OCH}_{3}\right), 6.37$ (d, 1H, $\left.\mathrm{H}_{\alpha}\right), 6.89$ (d, $\left.1 \mathrm{H}, \mathrm{H}_{\beta}\right), 6.92-8.10\left(\mathrm{~m}, 8 \mathrm{H}\right.$, aromatic), $11.13(\mathrm{~s}, 1 \mathrm{H}, \mathrm{OH}){ }^{13} \mathrm{C}-\mathrm{NMR}\left(\mathrm{CDCl}_{3}\right): \delta 55.5,58.4,114.8$, $122.9,126.8,127.4,127.6,127.8,131.1,143.1,156.8,160.3,169.8,197.2$. Mass (m/z): 324. Anal. (\%) for $\mathrm{C}_{18} \mathrm{H}_{16} \mathrm{~N}_{2} \mathrm{O}_{4}$, Calcd. C, 66.66; H, 4.97; N, 8.64; O, 19.73; Found: C, 66.55; H, 4.86; N, 8.52; O, 19.61 .

3-(3-Methoxy-4-hydroxy-phenyl)-1-[(4)-carboxyphenylazomethyl]-2-propene-1-one (2h). Yield 66\%, mp. 180-182 ${ }^{\circ} \mathrm{C}$, IR (KBr): $1153(\mathrm{C}-\mathrm{O}-\mathrm{C}), 1485(\mathrm{~N}=\mathrm{N}), 1716(\mathrm{C}=\mathrm{O}), 1801(\mathrm{C}=\mathrm{O}$ of acid), $3039(\mathrm{CH}) ; 3189(\mathrm{OH}) ;{ }^{1} \mathrm{H}-\mathrm{NMR}\left(\mathrm{DMSO}-d_{6}\right): \delta 3.10\left(\mathrm{~s}, 2 \mathrm{H}, \mathrm{CH}_{2}\right), 3.73\left(1 \mathrm{~N},-\mathrm{OCH}_{3}\right)$, $10.10(\mathrm{~s}, 1 \mathrm{H}, \mathrm{Ar}-\mathrm{OH}), 6.34\left(\mathrm{~d}, 1 \mathrm{H}, \mathrm{H}_{\alpha}\right), 6.78\left(\mathrm{~d}, 1 \mathrm{H}, \mathrm{H}_{\beta}\right), 6.92-8.1$ (m, 7H, aromatic), 11.13 (s, $1 \mathrm{H}, \mathrm{OH}) ;{ }^{13} \mathrm{C}-\mathrm{NMR}\left(\mathrm{CDCl}_{3}\right): \delta 56.2,58.4,112.9,116.7,120.4,122.9,126.6,127.8,129.4$, 131.9, 143.5, 145.3, 152.5, 157.4, 171.1, 197.3. Mass $(\mathrm{m} / \mathrm{z})$ : 370. Anal. (\%) for $\mathrm{C}_{18} \mathrm{H}_{16} \mathrm{~N}_{2} \mathrm{O}_{5}$, Calcd. C, 63.52; H, 4.74; N, 8.23; O, 23.51; Found: C, 63.40; H, 4.61; N, 8.11; O, 23.38.

3-(Styryl)-1-[(4)-carboxyphenylazomethyl]-2-propene-1-one (2i). Yield 64\%, mp. 207-209 ${ }^{\circ} \mathrm{C}$, IR (KBr): $1484(\mathrm{~N}=\mathrm{N}), 1690(\mathrm{C}=\mathrm{O}), 1718\left(\mathrm{C}=\mathrm{O}\right.$ of acid), $3038(\mathrm{CH}) ;{ }^{1} \mathrm{H}-\mathrm{NMR}\left(\mathrm{DMSO}-d_{6}\right)$ : $\delta 3.12\left(\mathrm{~s}, 2 \mathrm{H}, \mathrm{CH}_{2}\right), 6.57\left(\mathrm{~d}, 1 \mathrm{H}, \mathrm{H}_{\alpha}\right), 7.01\left(\mathrm{~d}, 1 \mathrm{H}, \mathrm{H}_{\beta}\right), 7.14-8.1(\mathrm{~m}, 9 \mathrm{H}$, aromatic), $11.03(\mathrm{~s}, 1 \mathrm{H}$, $\mathrm{OH}) ;{ }^{13} \mathrm{C}-\mathrm{NMR}\left(\mathrm{CDCl}_{3}\right): \delta 64.4,122.6,125.8,126.7,127.6,128.2,129.1,130.5,131.1,131.8$, 136.1, 142.8, 156.9, 170.6, 196.9. Mass (m/z): 320. Anal. (\%) for $\mathrm{C}_{19} \mathrm{H}_{16} \mathrm{~N}_{2} \mathrm{O}_{3}$, Calcd. C, 71.24; H, 5.03; N, 8.74; O, 14.98; Found: C, 71.12; H, 4.89; N, 8.61; O, 14.88 .

3-(2-Furyl)-1-[(4)-carboxyphenylazomethyl]-2-propene-1-one (2j). Yield 66\%, mp. 183-185 ${ }^{\circ} \mathrm{C}$, IR (KBr): $1155(\mathrm{C}-\mathrm{O}-\mathrm{C}), 1486(\mathrm{~N}=\mathrm{N}), 1687(\mathrm{C}=\mathrm{O}), 1707\left(\mathrm{C}=\mathrm{O}\right.$ of acid), $3044(\mathrm{CH}) ;{ }^{1} \mathrm{H}-$ NMR $\left(\mathrm{DMSO}-d_{6}\right): \delta 3.12\left(\mathrm{~s}, 2 \mathrm{H}, \mathrm{CH}_{2}\right), 6.67-7.54(\mathrm{dd}, 1 \mathrm{H},-\mathrm{CH}=\mathrm{CH}), 6.65-8.10(\mathrm{~m}, 7 \mathrm{H}$, 
aromatic), $11.90(\mathrm{~s}, 1 \mathrm{H}, \mathrm{OH}) ;{ }^{13} \mathrm{C}-\mathrm{NMR}\left(\mathrm{CDCl}_{3}\right): \delta 63.7,113.1,114.4,122.9,127.9,129.7$, 131.6, 132.9, 146.2, 151.9, 157.4, 170.8, 197.4. Mass $(\mathrm{m} / \mathrm{z})$ : 284. Anal. (\%) for $\mathrm{C}_{15} \mathrm{H}_{12} \mathrm{~N}_{2} \mathrm{O}_{4}$, Calcd. C, 63.38; H, 4.25; N, 9.85; O, 22.51; Found: C, 63.27; H, 4.13; N, 9.73; O, 22.38.

General method for the synthesis of 6-(4'-aryl)-4[(4)-carboxyphenylazomethyl]-2-hydroxyl1,6-dihydropyrimidine (3a-j)

A mixture of $0.01 \mathrm{~mol}$ of the required chalcone (2a-j), $0.01 \mathrm{~mol}$ urea and $\mathrm{KOH}$ (1gm) in $20 \mathrm{ml}$ ethanol was heated under reflux for 6 hours, then cooled and poured onto crushed ice. The soobtained solid product was filtered and recrystalized from ethanol.

6-(4'-Phenyl)-4[(4)-carboxyphenylazomethyl]-2-hydroxyl-1,6-dihydropyrimidine (3a). Yield 63\%, mp. $235-237{ }^{\circ} \mathrm{C}$, IR ( $\left.\mathrm{KBr}\right)$ : $1570(\mathrm{C}=\mathrm{C}$ ring skeleton Ar moiety), $1415(\mathrm{C}=\mathrm{C}$ ring skeleton pyrimidine moiety), $1480(\mathrm{~N}=\mathrm{N}), 1690\left(\mathrm{C}=\mathrm{O}\right.$ of acid), $3401(\mathrm{OH}) ;{ }^{1} \mathrm{H}-\mathrm{NMR}\left(\mathrm{DMSO}-d_{6}\right): \delta 2.44$ $\left(\mathrm{s}, 2 \mathrm{H}, \mathrm{CH}_{2}\right), 4.49(\mathrm{~s}, 1 \mathrm{H}, \mathrm{NH}-\mathrm{CH}), 4.90(\mathrm{~s}, 1 \mathrm{H}, \mathrm{OH}), 5.97(\mathrm{~d}, 1 \mathrm{H}, \mathrm{CH}), 6.61-7.96$ (m, 9H, Ar$\mathrm{H}), 12.32(\mathrm{~s}, 1 \mathrm{H}, \mathrm{OH}) ;{ }^{13} \mathrm{C}-\mathrm{NMR}\left(\mathrm{CDCl}_{3}\right): \delta 47.2,62.2,118.2,122.9,127.1,127.3,127.5,128.8$, 131.1, 139.6, 144.1, 156.7, 163.4, 170.1. Mass (m/z): 336. Anal. (\%) for $\mathrm{C}_{18} \mathrm{H}_{16} \mathrm{~N}_{4} \mathrm{O}_{3}$, Calcd. C, 64.28; H, 4.79; N, 16.66; O, 14.27. Found: C, 64.28; H, 4.79; N, 16.66; O, 14.27.

6-(2'-Hydroxyphenyl)-4[(4)-carboxyphenylazomethyl]-2-hydroxyl-1,6dihydropyrimidine (3b). Yield 70\%, mp. 212-214 ${ }^{\circ} \mathrm{C}$, IR (KBr): 1568 ( $\mathrm{C}=\mathrm{C}$ ring skeleton Ar moiety), $1411(\mathrm{C}=\mathrm{C}$ ring skeleton pyrimidine moiety), $1483(\mathrm{~N}=\mathrm{N}), 1688(\mathrm{C}=\mathrm{O}$ of acid), $3210(\mathrm{~N}-\mathrm{H}), 3409(\mathrm{OH})$; ${ }^{1} \mathrm{H}-\mathrm{NMR}\left(\mathrm{DMSO}-d_{6}\right): \delta 2.49\left(2 \mathrm{H}, s, \mathrm{CH}_{2}\right), 4.47(\mathrm{~d}, 1 \mathrm{H}, \mathrm{NH}-\mathrm{CH}), 4.88(\mathrm{~s}, 1 \mathrm{H}, \mathrm{CH}), 5.29(\mathrm{~s}, 1 \mathrm{H}$, $\mathrm{NH}), 5.89(\mathrm{~d}, 1 \mathrm{H}, \mathrm{CH}), 6.64-7.90(\mathrm{~m}, 8 \mathrm{H}, \mathrm{Ar}-\mathrm{H}), 11.90(\mathrm{~s}, 1 \mathrm{H}, \mathrm{OH}), 12.39\left(\mathrm{~s}, 1 \mathrm{H}, \mathrm{OH}_{\mathrm{acid}}\right) ;{ }^{13} \mathrm{C}-$ NMR $\left(\mathrm{CDCl}_{3}\right): \delta 37.2,62.6,115.9,117.9,121.6,12.7,123,127.9,128.3,128.9,131.3,139.9$, 154,7, 156.6, 163.9, 171.1. Mass (m/z): 352. Anal. (\%) for $\mathrm{C}_{18} \mathrm{H}_{16} \mathrm{~N}_{4} \mathrm{O}_{4}$, Calcd. C C, 61.36; H, 4.58; N, 15.90; O, 18.16; Found: C, 61.36; H, 4.58; N, 15.90; O, 18.16.

6-(4'-Hydroxyphenyl)-4[(4)-carboxyphenylazomethyl]-2-hydroxyl-1,6-dihydropyrimidine (3c). Yield 72\%, mp. 249-251 ${ }^{\circ} \mathrm{C}$, IR $(\mathrm{KBr})$ : $1556(\mathrm{C}=\mathrm{C}$ ring skeleton Ar. moiety), $1418(\mathrm{C}=\mathrm{C}$ ring skeleton pyrimidine moiety), $1479(\mathrm{~N}=\mathrm{N}), 1692(\mathrm{C}=\mathrm{O}$ of acid), $3210(\mathrm{~N}-\mathrm{H}), 3412(\mathrm{OH})$; ${ }^{1} \mathrm{H}-\mathrm{NMR}\left(\mathrm{DMSO}-d_{6}\right): \delta 2.47\left(\mathrm{~s}, 2 \mathrm{H}, \mathrm{CH}_{2}\right), 4.46(\mathrm{~d}, 1 \mathrm{H}, \mathrm{NH}-\mathrm{C} \underline{\mathrm{H}}), 4.86(\mathrm{~s}, 1 \mathrm{H}, \mathrm{OH}), 5.24(\mathrm{~s}, 1 \mathrm{H}$, $\mathrm{NH}), 5.90(\mathrm{~d}, 1 \mathrm{H}, \mathrm{CH}), 6.60-7.96(\mathrm{~m}, 8 \mathrm{H}, \mathrm{Ar}-\mathrm{H}), 11.88(\mathrm{~s}, 1 \mathrm{H}, \mathrm{OH}), 12.29\left(\mathrm{~s}, 1 \mathrm{H}, \mathrm{OH}_{\mathrm{acid}}\right) ;{ }^{13} \mathrm{C}-$ NMR ( $\left.\mathrm{CDCl}_{3}\right): \delta 47.2,62.6,112.9,114.1,117.9,120.1,123.1,127.8,130.9,131.1,139.6,144.9$, 156.4, 163.7, 170.2. Mass (m/z): 352. Anal. (\%) for $\mathrm{C}_{18} \mathrm{H}_{16} \mathrm{~N}_{4} \mathrm{O}_{4}$, Calcd. C, 61.36; H, 4.58; N, 15.90; O, 18.16; Found: C, 61.36; H, 4.58; N, 15.90; O, 18.16.

6-(2'-Nitrophenyl)-4[(4)-carboxyphenylazomethyl]-2-hydroxyl-1,6-dihydropyrimidine (3d). Yield 76\%, mp. 227-229 ${ }^{\circ} \mathrm{C}$, IR (KBr): 1563 (C=C ring skeleton Ar. moiety), $1380\left(\mathrm{NO}_{2}\right), 1412$ $(\mathrm{C}=\mathrm{C}$ ring skeleton pyrimidine moiety), $1483(\mathrm{~N}=\mathrm{N}), 1698(\mathrm{C}=\mathrm{O}$ of acid), $3209(\mathrm{~N}-\mathrm{H}), 3402$ $(\mathrm{OH}) ;{ }^{1} \mathrm{H}-\mathrm{NMR}$ (DMSO-d $\left.)_{6}\right): \delta 2.42\left(\mathrm{~s}, 2 \mathrm{H}, \mathrm{CH}_{2}\right), 4.47(\mathrm{~d}, 1 \mathrm{H}, \mathrm{NH}-\mathrm{CH}), 4.80(\mathrm{~s}, 1 \mathrm{H}, \mathrm{OH}), 5.26$ (s, 1H, NH), $5.91(\mathrm{~d}, 1 \mathrm{H}, \mathrm{CH}), 7.11-8.92(\mathrm{~m}, 8 \mathrm{H}, \mathrm{Ar}-\mathrm{H}), 12.19(\mathrm{~s}, 1 \mathrm{H}, \mathrm{OH}) ;{ }^{13} \mathrm{C}-\mathrm{NMR}\left(\mathrm{CDCl}_{3}\right)$ : $\delta 38.4,63.6,118.4,121.4,122.8,127.4,127.9,131.2,134.6,138.3,139.4,148.1,156.9,163.9$, 172.1. Mass (m/z): 381. Anal. (\%) for $\mathrm{C}_{18} \mathrm{H}_{15} \mathrm{~N}_{5} \mathrm{O}_{5}$, Calcd. C, 56.69; H, 3.96; N, 18.37; O, 20.98; Found: C, 56.69; H, 3.96; N, 18.37; O, 20.98. 
6-(3'-Nitrophenyl)-4[(4)-carboxyphenylazomethyl]-2-hydroxyl-1,6-dihydropyrimidine (3e). Yield 68\%, mp. 279-281 ${ }^{\circ} \mathrm{C}$, IR (KBr): 1565 (C=C ring skeleton Ar. moiety), $1378\left(\mathrm{NO}_{2}\right), 1411$ $(\mathrm{C}=\mathrm{C}$ ring skeleton pyrimidine moiety), $1478(\mathrm{~N}=\mathrm{N}), 1694(\mathrm{C}=\mathrm{O}$ of acid), $3212(\mathrm{~N}-\mathrm{H}), 3412$ $(\mathrm{OH}) ;{ }^{1} \mathrm{H}-\mathrm{NMR}\left(\mathrm{DMSO}-d_{6}\right): \delta 2.39\left(\mathrm{~s}, 2 \mathrm{H}, \mathrm{CH}_{2}\right), 4.46$ (d, 1H, NH-C프), 4.79 (s, 1H, OH), 5.21 $(\mathrm{s}, 1 \mathrm{H}, \mathrm{NH}), 5.90(\mathrm{~d}, 1 \mathrm{H}, \mathrm{CH}), 7.08-8.42(\mathrm{~m}, 8 \mathrm{H}, \mathrm{Ar}-\mathrm{H}), 12.21(\mathrm{~s}, 1 \mathrm{H}, \mathrm{OH}) ;{ }^{13} \mathrm{C}-\mathrm{NMR}\left(\mathrm{CDCl}_{3}\right)$ : $\delta 46.2,62.8,118.2,119.3,122.6,122.9,128.2,130.2,131.6,133.0,139.9,144.9,149.2,156.8$, 164.2, 169.8. Mass (m/z): 381. Anal. (\%) for $\mathrm{C}_{18} \mathrm{H}_{15} \mathrm{~N}_{5} \mathrm{O}_{5}$, Calcd. C, 56.69; H, 3.96; N, 18.37; O, 20.98; Found: C, 56.69; H, 3.96; N, 18.37; O, 20.98.

6-(2'-Chlorophenyl)-4[(4)-carboxyphenylazomethyl]-2-hydroxyl-1,6-dihydropyrimidine (3f). Yield 65\%, mp. 251-253 ${ }^{\circ} \mathrm{C}$, IR (KBr): $756(\mathrm{Cl}), 1561(\mathrm{C}=\mathrm{C}$ ring skeleton Ar. moiety), 1415 $(\mathrm{C}=\mathrm{C}$ ring skeleton pyrimidine moiety), $1486(\mathrm{~N}=\mathrm{N}), 1696(\mathrm{C}=\mathrm{O}$ of acid), $3208(\mathrm{~N}-\mathrm{H}), 3412$ $(\mathrm{OH}) ;{ }^{1} \mathrm{H}-\mathrm{NMR}\left(\mathrm{DMSO}-d_{6}\right): \delta 2.44\left(\mathrm{~s}, 2 \mathrm{H}, \mathrm{CH}_{2}\right), 4.49$ (d, 1H, NH-C프), 4.90 (s, 1H, OH), 5.24 $(\mathrm{s}, 1 \mathrm{H}, \mathrm{NH}), 5.94(\mathrm{~d}, 1 \mathrm{H}, \mathrm{CH}), 7.11-8.36(\mathrm{~m}, 8 \mathrm{H}, \mathrm{Ar}-\mathrm{H}), 12.29(\mathrm{~s}, 1 \mathrm{H}, \mathrm{OH}) ;{ }^{13} \mathrm{C}-\mathrm{NMR}\left(\mathrm{CDCl}_{3}\right)$ : $\delta 37.9,62.1,118.1,123.1,126.9,127.6,128.3,128.6,128.9,131.4,132.7,139.4,143.4,156.9$, 163.6, 170.6. Mass (m/z): 370. Anal. (\%) for $\mathrm{C}_{18} \mathrm{H}_{15} \mathrm{ClN}_{4} \mathrm{O}_{3}$, Calcd. C, 58.31; H, 4.08; Cl, 9.56; N, 15.11; O, 12.94; Found: C, 58.31; H, 4.08; Cl, 9.56; N, 15.11; O, 12.94.

6-(4'-Methoxyphenyl)-4[(4)-carboxyphenylazomethyl]-2-hydroxyl-1,6-dihydropyrimidine (3g). Yield 73\%, mp. 219-221 ${ }^{\circ} \mathrm{C}$, IR ( $\left.\mathrm{KBr}\right): 1560(\mathrm{C}=\mathrm{C}$ ring skeleton Ar. moiety), $1411(\mathrm{C}=\mathrm{C}$ ring skeleton pyrimidine moiety), $1487(\mathrm{~N}=\mathrm{N}), 1693(\mathrm{C}=\mathrm{O}$ of acid), $3210(\mathrm{~N}-\mathrm{H}), 3400(\mathrm{OH})$; ${ }^{1} \mathrm{H}-\mathrm{NMR}\left(\mathrm{DMSO}-d_{6}\right): \delta 2.34\left(\mathrm{~s}, 2 \mathrm{H}, \mathrm{CH}_{2}\right), 3.72\left(\mathrm{~s}, 1 \mathrm{H}, \mathrm{OCH}_{3}\right), 4.46(\mathrm{~d}, 1 \mathrm{H}, \mathrm{NH}-\mathrm{C} \underline{\mathrm{H}}), 4.85$ (s, $1 \mathrm{H}, \mathrm{OH}), 5.19(\mathrm{~s}, 1 \mathrm{H}, \mathrm{NH}), 5.92(\mathrm{~d}, 1 \mathrm{H}, \mathrm{CH}), 6.42-8.06(\mathrm{~m}, 8 \mathrm{H}, \mathrm{Ar}-\mathrm{H}), 12.32(\mathrm{~s}, 1 \mathrm{H}, \mathrm{OH}) ;{ }^{13} \mathrm{C}-$ NMR (CDCl3): $\delta 46.9,56.4,62.3,114.9,117.9,122.8,127.6,128.7,131.4,136.3,140.3,156.4$, 159.2, 163.9, 169.8. Mass (m/z): 366. Anal. (\%) for $\mathrm{C}_{19} \mathrm{H}_{18} \mathrm{~N}_{4} \mathrm{O}_{4}$, Calcd. C, 62.29; H, 4.95; N, 15.29; O, 17.47; Found: C, 62.29; H, 4.95; N, 15.29; O, 17.47.

6-(3-Methoxy-4-hydroxy-phenyl)-4[(4)-carboxyphenylazomethyl]-2-hydroxyl-1,6-dihydropyrimidine (3h). Yield 75\%, mp. 241-243 ${ }^{\circ} \mathrm{C}$, IR ( $\left.\mathrm{KBr}\right) 1559$ (C=C ring skeleton Ar. moiety), $1410(\mathrm{C}=\mathrm{C}$ ring skeleton pyrimidine moiety), $1489(\mathrm{~N}=\mathrm{N}), 1696(\mathrm{C}=\mathrm{O}$ of acid), $3209(\mathrm{~N}-\mathrm{H})$, $3412(\mathrm{OH}) ;{ }^{1} \mathrm{H}-\mathrm{NMR}\left(\mathrm{DMSO}-d_{6}\right): \delta 2.49\left(\mathrm{~s}, 2 \mathrm{H}, \mathrm{CH}_{2}\right), 3.69\left(\mathrm{~s}, 1 \mathrm{H}, \mathrm{OCH}_{3}\right), 4.47$ (d, 1H, NH$\mathrm{C} \underline{\mathrm{H}}), 4.81(\mathrm{~s}, 1 \mathrm{H}, \mathrm{OH}), 5.14(\mathrm{~s}, 1 \mathrm{H}, \mathrm{NH}), 5.95(\mathrm{~d}, 1 \mathrm{H}, \mathrm{CH}), 6.59-7.96(\mathrm{~m}, 7 \mathrm{H}, \mathrm{Ar}-\mathrm{H}), 11.84(\mathrm{~s}$, $1 \mathrm{H}, \mathrm{OH}), 12.39(\mathrm{~s}, 1 \mathrm{H}, \mathrm{OH}) ;{ }^{13} \mathrm{C}-\mathrm{NMR}\left(\mathrm{CDCl}_{3}\right): \delta 47.5,56.9,62.3,112.7,116.9,117.5,120.9$, 122.8, 127.7, 131.2, 137.4, 139.9, 144.2, 151.9, 157.3, 163.6, 170.9. Mass (m/z): 382. Anal. (\%) for $\mathrm{C}_{19} \mathrm{H}_{18} \mathrm{~N}_{4} \mathrm{O}_{5}$, Calcd. C, 59.68; H, 4.74; N, 14.65; O, 20.92; Found: C, 59.68; H, 4.74; N, 14.65; O, 20.92.

6-(Styryl)-4[(4)-carboxyphenylazomethyl]-2-hydroxyl-1,6-dihydropyrimidine (3i). Yield 66\%, mp. 244-246 ${ }^{\circ} \mathrm{C}$, IR (KBr) $1554(\mathrm{C}=\mathrm{C}$ ring skeleton Ar. moiety), $1423(\mathrm{C}=\mathrm{C}$ ring skeleton pyrimidine moiety), $1486(\mathrm{~N}=\mathrm{N}), 1699\left(\mathrm{C}=\mathrm{O}\right.$ of acid), $3411(\mathrm{OH}) ;{ }^{1} \mathrm{H}-\mathrm{NMR}\left(\mathrm{DMSO}-d_{6}\right): \delta 2.48$ $\left(\mathrm{s}, 2 \mathrm{H}, \mathrm{CH}_{2}\right), 4.18(\mathrm{~d}, 1 \mathrm{H}, \mathrm{NH}-\mathrm{CH}), 4.79(\mathrm{~s}, 1 \mathrm{H}, \mathrm{OH}), 5.21(\mathrm{~s}, 1 \mathrm{H}, \mathrm{NH}), 6.12(\mathrm{~d}, 1 \mathrm{H}, \mathrm{CH}), 6.29$ (dd, 1H, CH), 6.64 (d, 2H, CH), 7.14-8.31 (m, 8H, Ar-H), $12.40(\mathrm{~s}, 1 \mathrm{H}, \mathrm{OH}) ;{ }^{13} \mathrm{C}-\mathrm{NMR}\left(\mathrm{CDCl}_{3}\right)$ : $\delta 49.6,62.7,118.2,123.1,123.7,126.7,127.8,128.2,128.9,129.7,131.2,140.0,157.4,163.5$, 
170.7. Mass $(\mathrm{m} / \mathrm{z})$ : 362. Anal. (\%) for $\mathrm{C}_{20} \mathrm{H}_{18} \mathrm{~N}_{4} \mathrm{O}_{3}$, Calcd. C, 66.29; $\mathrm{H}, 5.01 ; \mathrm{N}, 15.46 ; \mathrm{O}, 13.25$; Found: C, 66.29; H, 5.01; N, 15.46; O, 13.25 .

6-(2-Furyl)-4[(4)-carboxyphenylazomethyl]-2-hydroxyl-1,6-dihydropyrimidine (3j). Yield 64\%, mp. 236-238 ${ }^{\circ} \mathrm{C}$, IR ( $\left.\mathrm{KBr}\right) 1569(\mathrm{C}=\mathrm{C}$ ring skeleton Ar. moiety), $1418(\mathrm{C}=\mathrm{C}$ ring skeleton

pyrimidine moiety), $1489(\mathrm{~N}=\mathrm{N}), 1701\left(\mathrm{C}=\mathrm{O}\right.$ of acid), $3210(\mathrm{~N}-\mathrm{H}), 3404(\mathrm{OH}) ;{ }^{1} \mathrm{H}-\mathrm{NMR}$ (DMSO- $\left.d_{6}\right): \delta 2.39$ (s, 2H, CH $), 4.49(\mathrm{~d}, 1 \mathrm{H}, \mathrm{NH}-\mathrm{C} \underline{\mathrm{H}}), 4.84(\mathrm{~s}, 1 \mathrm{H}, \mathrm{OH}), 5.14(\mathrm{~s}, 1 \mathrm{H}, \mathrm{NH}), 5.89$ (d, $1 \mathrm{H}, \mathrm{CH}), 6.32-8.29\left(\mathrm{~m}, 7 \mathrm{H}, \mathrm{Ar}-\mathrm{H}\right.$, furyl), $12.30(\mathrm{~s}, 1 \mathrm{H}, \mathrm{OH}) ;{ }^{13} \mathrm{C}-\mathrm{NMR}\left(\mathrm{CDCl}_{3}\right): \delta 48.2,62.3$, 107.3, 111.3, 118.6, 122.7, 127.6, 131.2, 139.8, 142.0, 153.6, 157.4, 163.7, 171.6. Mass $(\mathrm{m} / \mathrm{z})$ : 326. Anal. (\%) for $\mathrm{C}_{16} \mathrm{H}_{14} \mathrm{~N}_{4} \mathrm{O}_{4}$, Calcd. C, 58.89; H, 4.32; N, 17.17; O, 19.61; Found: C, 58.89; $\mathrm{H}, 4.32 ; \mathrm{N}, 17.17 ; \mathrm{O}, 19.61$.

\section{Acknowledgements}

The authors wish to thank the Professor and Head, Department of Chemistry Saurashtra University, Rajkot for research facilities. Authors are thankful to CDRI Lucknow for spectral and analytical data. We thank the Tuberculosis Antimicrobial Acquisition and Coordinating Faculty (TAACF), www.taacf.org for the in vitro antimycobacterial screening.

\section{References}

1. http://www.who.int/tdr/diseases/tb/default.htm.

2. Dhar, D. N. The Chemistry of Chalcones and Related Compounds, Wiley: New York, 1981; p 5 .

3. Raut, K. B.; Wendor, D. H. J. Org. Chem. 1960, 25, 2126.

4. Ariyan, Z. S.; Suschitzky, H. J. J. Chem. Soc. 1961, 2242.

5. Jurasek, A.; Knoppava, V.; Dandarova, M.; Kovac, A.; Reinprecht, J. Tetrahedron 1978, 34, 1883.

6. Subbraju, G. V.; RangaNayakulu, A.; Parameshwara, D. Indian J. Heterocyclic Chem. 1994, $4,87$.

7. Bhat, B. A.; Dhar, K. L.; Puri, S. C.; Saxena, A. K.; Shanmugavel, M.; Qazi, G. N. Bioorg. Med. Chem. Lett. 2005, 15, 3177.

8. Domínguez, J. N.; Rodrigues,C. J.; Gamboa de Domínguez, N.; Gut, J.; Philip, J.; Rosenthal, P. J. Farmaco 2005, 60, 307.

9. Yaulì, N.; Üçüncü, O.; Aydìn, E.; Gök, Y.; Yaar, A.; Baltacì, C.; Yìldìrìm, N.; Küçük, M. J. Photochem. Photobiol. A: Chem. 2005, 169, 229.

10. Khatib, S.; Narya, O.; Musa, R.; Shmuel, M.; Tamir, S.; Vaya, J. Bioorg. Med. Chem. 2005, $13,433$.

11. Nigam, S. C.; Saharia, G. S.; Sharma, H. R. Indian Chem. Soc. 1983, 60, 583 
12. Baddiley, J.; Lythgoe, B.; Todd, A. R. J. Chem. Soc. 1944, 318.

13. Kenner, G. W.; Lythoge, B.; Todd, A. R. J. Chem. Soc. 1944, 652.

14. Centolella, A.P.; Nelson, J. W.; Kolloff, H. G. J. Am. Chem. Soc. 1943, 65, 209.

15. Noriyuki, K.; Hitoshi, M.; Shionogi \& Co. Ltd., Japan PCT Int. Appl. WO 03, 47, 564, 2002; Chem. Abstr. 2003, 139, 36532c.

16. Jani, M. K.; Shah, B. R.; Undavia, N. K.; Trivedi, P. B. Chem. Abstr. 1994, 121, 35513p.

17. Safonova, T.V.; Keremov, A.F.; Ershova, Yu. A. Khim. Farm. Zn. 1998, 32 (12), 11, (Eng); Chem. Abstr. 1999, 131, 18975e.

18. Jean-Damien, C.; David, B.; Ronald, K.; Julian, G.; Pan, Li; Robert, D.; Vertex Pharmaceuticals Incorporated, USA; PCT Int. Appl. WO 02 22, 608, 2002; Chem. Abstr. 2002, 136, 247584x.

19. Nakaguti, O.; Shimazaki, N.; Shimazaki, M.; Nakatuka, M., Eur. Pat. Appl., 168, 005, 1986; Chem. Abstr. 1986, 105, 191118p.

20. Papesh, V.; Schroeder, E. F. US. Pat 2714559, 1956: Chem. Abstr. 1956, 50, 11370.

21. Tokutake, N.; Brit. Pat.146836B, 1977; Chem. Abstr. 1977, 87, 102370.

22. Kurono M.; JP, 62, 267, 272, 1987; Chem. Abstr. 1988, 109, 37382t.

23. Ojha, A. C.; Jain, R. Polish J. Chem. 1982, 56, 1553.

24. Franzblau, S. G.; Witzig, R. S.; McLaughlin, J. C.; Torres, P.; Madico, G.; Hernandez, A.; Degnan, M. T.; Cook, M. B.; Quenzer, V. K.; Ferguson, R. M.; Gilman, R. H. J. Clin. Microbiol. 1998, 36, 362.

25. Vanitha, J. D.; Paramasivan, C. N. Mycobacteriology 2004, 49, 179.

26. Reis, R. S.; Neves, I. S.; Lourenco, L. S.; Fonseca, L. S.; Lourenco, M. C. S. J. Clin. Microbiol. 2004, 42, 2247. 